\title{
Kinetic Monte Carlo Simulations of Oxygen Diffusion in Environmental Barrier Coating Materials
}

Brian S. Good

NASA Glenn Research Center, Cleveland, OH 44135

\begin{abstract}
Ceramic Matrix Composite (CMC) materials are of interest for use in next-generation turbine engine components, offering a number of significant advantages, including reduced weight and high operating temperatures. However, in the hot environment in which such components operate, the presence of water vapor can lead to corrosion and recession, limiting the useful life of the components. Such degradation can be reduced through the use of Environmental Barrier Coatings (EBCs) that limit the amount of oxygen and water vapor reaching the component. Candidate EBC materials include Yttrium and Ytterbium silicates. In this work we present results of kinetic Monte Carlo (kMC) simulations of oxygen diffusion, via the vacancy mechanism, in Yttrium and Ytterbium disilicates, along with a brief discussion of interstitial diffusion.

An EBC system typically includes a bond coat located between the EBC and the component surface. Bond coat materials are generally chosen for properties other than low oxygen diffusivity, but low oxygen diffusivity is nevertheless a desirable characteristic, as the bond coat could provide some additional component protection, particularly in the case where cracks in the coating system provide a direct path from the environment to the bond coat interface. We have therefore performed similar kMC simulations of oxygen diffusion in this material.
\end{abstract}

\section{INTRODUCTION}

Ceramic Matrix Composite (CMC) materials are under consideration for use in nextgeneration jet turbine engines. Such materials offer high melting temperatures, light weight and good high-temperature strength, permitting increased operating temperatures and increased efficiencies. However, such components operate in an environment characterized not only by high temperatures, but by the presence of water vapor. Such an environment can lead to rapid corrosion, recession and failure, unless steps are taken to protect the vulnerable components.

Ceramics coating systems, typically consisting of an Environmental Barrier Coating (EBC), a Thermal Barrier Coating (TBC), and a bond coat, are being developed to address this issue ${ }^{1}$. Ceramics such as $\mathrm{Y}$ and $\mathrm{Yb}$ mono and disilicates are candidate materials for use in the EBC component of such systems, and the diffusive transport of water vapor and oxygen through these materials is of concern. In an effort to understand this diffusive process. we have performed atomistic simulations of oxygen diffusivity, for $\beta-\mathrm{Yb}_{2} \mathrm{Si}_{2} \mathrm{O}_{7}$, and $\delta$ - and $\gamma-\mathrm{Y}_{2} \mathrm{Si}_{2} \mathrm{O}_{7}$, using the kinetic Monte Carlo (kMC) method ${ }^{2}$. The migration barrier energies needed for the kMC simulations were computed using Density Functional Theory (DFT) ${ }^{3}$. While bond coat materials are typically chosen for reasons other than low oxygen diffusivity, a low-diffusivity bond coat could offer some degree of addition protection from corrosion. We have therefore performed similar kMC simulations for hafnium silicate, $\mathrm{HfSiO}_{4}$, a proposed bond coat material. Structural information for high-temperature phases of all four materials is shown in Table 1. 
Table 1. Structural information for candidate coating materials.

\begin{tabular}{|l|l|l|l|l|l|l|}
\hline Name & Crystal type & $\begin{array}{l}\text { Space } \\
\text { group }\end{array}$ & $\begin{array}{l}\text { Atoms } \\
\text { per cell }\end{array}$ & $\begin{array}{l}\text { Distinct } \\
\text { oxygen } \\
\text { sites }\end{array}$ & $a($ expt $), \AA$ & $a($ DFT $), \AA$ \\
\hline$\beta-\mathrm{Yb}_{2} \mathrm{Si}_{2} \mathrm{O}_{7}$ & Monoclinic & $\mathrm{C} 2 / \mathrm{m}$ & 22 & 3 & 6.802 & 6.825 \\
\hline$\gamma-\mathrm{Y}_{2} \mathrm{Si}_{2} \mathrm{O}_{7}$ & Monoclinic & $\mathrm{P}{ }_{1} / \mathrm{C}$ & 22 & 4 & 4.688 & 4.731 \\
\hline$\delta-\mathrm{Y}_{2} \mathrm{Si}_{2} \mathrm{O}_{7}$ & Orthorhombic & $\mathrm{Pna}_{1}$ & 44 & 7 & 13.68 & 13.797 \\
\hline $\mathrm{HfSiO}_{4}$ & Tetragonal & $\mathrm{I} 4{ }_{1} / \mathrm{amd}$ & 24 & 1 & 6.573 & 6.609 \\
\hline
\end{tabular}

\section{THEORY}

The kinetic Monte Carlo method is aimed at the study of so-called "infrequent-event" systems--those in which the events of interest are temporally separated from each other by long periods during which no such events take place ${ }^{2}$. While molecular dynamics (MD) is the method of choice for the study of many materials, it is ill-suited for infrequent-event systems, as a large fraction of computational resources are expended computing atomic trajectories between events. In such cases, MD may be many orders of magnitude less efficient than $\mathrm{kMC}$. The $\mathrm{kMC}$ method does not provide the detailed atomic trajectories available from MD simulations, but does produce trajectories that are statistically equivalent to those from $\mathrm{MD}$, and can be used to calculate certain properties very efficiently.

Diffusive hopping is an example of an infrequent-event process; at all but the lowest temperatures it can be considered a thermally activated process that proceeds via the hopping of atoms among crystalline and interstitial sites ${ }^{4}$. The kinetic Monte Carlo method has been used to simulate oxygen diffusion in other ceramic materials, notably yttria-stabilized zirconia, and we employ a similar method here 5 .

Very briefly, a kMC diffusion simulation follows the trajectories of a series of random walkers, representing diffusing atoms, traversing a lattice, with each atomic site possessing a number of potential hopping paths. When the hops are statistically independent, the hopping rate for each path is given by $v_{A B}=v^{0} \exp \left(-E_{A B} / k_{B} T\right)$, in which $v_{A B}$ and $E_{A B}$ are the hopping rate and migration barrier energy, respectively, and $v^{0}$ is a frequency factor representative of atomic vibrational frequencies. $v^{0}$ is typically assumed to be on the order of $10^{13} \mathrm{~s}^{-1}$. When all hopping rates have been calculated, the hopping probability for each path is calculated as $P_{A B}=v_{A B} / \Gamma$, in which $\Gamma$ is the sum of hopping rates for all hops accessible to the system. When the hopping probabilities are known for all paths, one of the accessible hops is chosen stochastically and executed, with the diffusing atom moving to a nearby vacancy or interstitial site. The execution of the hop exposes a number of newly-accessible hops, and renders some of the previously accessible ones no longer accessible, and the list of accessible hops is updated to incorporate the changes. The simulation clock is advanced stochastically by an amount consistent with the mean time between hops, $\Delta t=-\ln (R) / \Gamma$, with $0<R \leq 1$ a random number. When a sufficient number 
of hops have been executed, the mean square displacement $\left\langle\mathrm{R}^{2}>\right.$ is computed, and the diffusivity obtained from the Einstein relation $<\mathrm{R}^{2}>=6 \mathrm{D}$ t. A detailed description of the method is provided by Voter $^{2}$.

Our kMC simulations were performed using a computer code developed in our laboratory. Each incorporated $4 \times 10^{11}$ diffusive hops. For kMC simulations of vacancy-mechanism oxygen diffusion, it is convenient to track the motions of the relatively small number of vacancies, rather than the much larger number of oxygen atoms. This procedure yields the vacancy diffusivity, and the oxygen diffusivity can be obtained by distributing the vacancy displacements over the more numerous oxygen atoms by multiplying the vacancy diffusivity by a concentration correction factor $\mathrm{C}_{\mathrm{v}} /\left(1-\mathrm{C}_{\mathrm{v}}\right)$, in which $\mathrm{C}_{\mathrm{v}}$ is the vacancy concentration.

\section{DISCUSSION}

All ab initio calculations were performed using density functional theory (DFT) incorporating the plane-wave pseudopotential scheme and the Projector Augmented Wave approach $^{6,7}$, as implemented in the VASP computer code ${ }^{8}$. The calculations used functionals incorporating the Generalized Gradient Approximation of Perdew, Burke and Ernzerhof ${ }^{9}$. A large number of potential diffusion paths were investigated, and $1 \times 1 \times 1$ cells were used to reduce computation time. $1 \times 1 \times 1$ cells for all materials considered here are fairly large, containing at least 22 atoms, and a limited number of calculations using $2 \times 2 \times 2$ cells suggest that the $1 \times 1 \times 1$ cells are of adequate size to give reasonably accurate relaxations and barrier energies.

Lattice constants $a$ were optimized while constraining $b / a, c / a$ and all angles to their experimental values. As shown in Table 1, the values obtained for $a$ are slightly larger than the experimental values, consistent with the Generalized Gradient Approximation's tendency to underbind.

\section{$\underline{\text { Vacancy-mechanism diffusion }}$}

Vacancy formation energies were computed, and the ranges of values for all materials are shown in Table 2. Note that the values for $\mathrm{Yb}$ disilicate are smaller than those for the other materials, suggesting that the intrinsic vacancy concentration will be larger in that material. However, all vacancy formation energies are large enough that, for the range of temperatures considered here, the intrinsic vacancy concentrations for all materials will be very small, as will the concentration correction factors.

Migration barrier energies for oxygen diffusion via the vacancy mechanism were computed using the Improved Dimer Method ${ }^{10}$ included in the Transition State Tools suite of codes from the University of Texas ${ }^{11}$. Space limitations preclude a listing of all barrier energies for all materials, but the ranges of energies for each material are shown in Table 2. It can be seen that $\mathrm{Yb}$ disilicate has the smallest barrier energies.

Vacancy-mechanism oxygen diffusivities from $\mathrm{kMC}$ simulations, as functions of temperature, are shown in Figure 1. It can be seen that the diffusivities are largest for $\mathrm{Yb}$ disilicate; those of the two Y disilicate structures are substantially smaller. The presence of a small barrier $(0.46 \mathrm{eV})$ for $\mathrm{Yb}$ disilicate is a partial explanation for this, but the vacancy formation energies are considerably smaller, as well, and consequently the concentration correction factors for $\mathrm{Yb}$ disilicate are considerably larger. 


\begin{tabular}{|l|l|l|}
\hline Name & $\begin{array}{l}\text { Vacancy formation } \\
\text { energy range, eV }\end{array}$ & $\begin{array}{l}\text { Barrier energy } \\
\text { range, eV }\end{array}$ \\
\hline$\beta-\mathrm{Yb}_{2} \mathrm{Si}_{2} \mathrm{O}_{7}$ & $2.63-3.72$ & $0.46-8.39$ \\
\hline$\gamma-\mathrm{Y}_{2} \mathrm{Si}_{2} \mathrm{O}_{7}$ & $5.15-5.48$ & $1.80-8.15$ \\
\hline$\delta-\mathrm{Y}_{2} \mathrm{Si}_{2} \mathrm{O}_{7}$ & $5.03-5.49$ & $0.97-4.53$ \\
\hline $\mathrm{HfSiO}_{4}$ & 4.56 & $2.39-11.81$ \\
\hline
\end{tabular}

Table 2. Vacancy formation energies and vacancy-mechanism migration barrier energy ranges

The bond coat material, $\mathrm{HfSiO}_{4}$, exhibits a range of barriers that is somewhat larger than those of the two Y disilicate polytypes, which should reduce the diffusivity. However, the vacancy formation energy lies below the ranges of the two Ydisilicates, which serves to increase the diffusivity. The net result is a diffusivity curve that is very similar to those of the $\mathrm{Y}$ disilicates, suggesting that the bond coat can in fact offer a degree of additional protection.

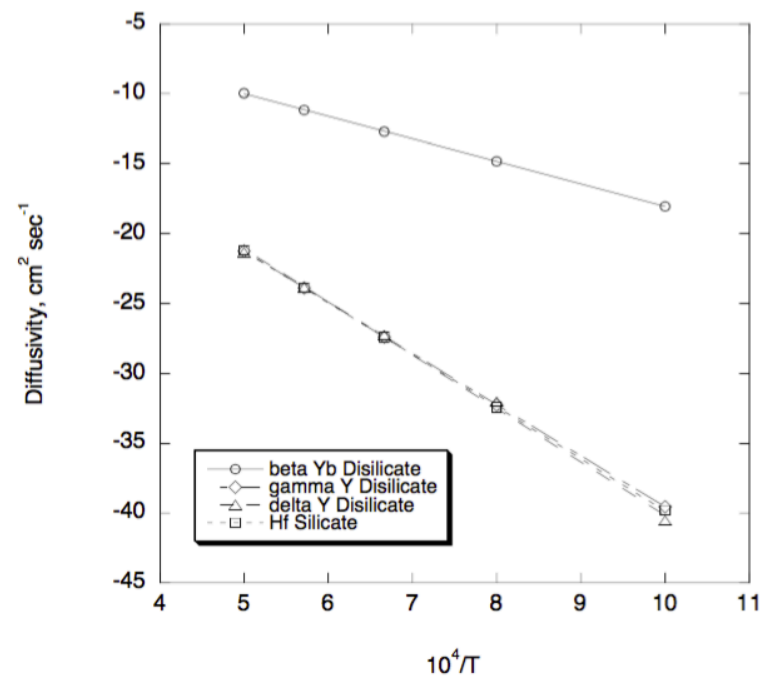

Figure 1. Vacancy-mechanism oxygen diffusivity

It should be noted that the above diffusivities were computed under the assumption that only intrinsic vacancies were present. However, other candidate coating materials, for example, Yttria-stabilized Zirconia (YSZ), can contain large concentrations of extrinsic vacancies induced by the charge mismatch between the $\mathrm{Y}$ and $\mathrm{Zr}$ cations, leading to a much higher vacancymechanism diffusivity. A major concern with respect to the utility of the materials included in 
this study is that such coatings are vulnerable to attack by reactive glasses, for example, calcium magnesium aluminosilicate (CMAS) ${ }^{12}$. If $\mathrm{Ca}$ ions are incorporated substitutionally into the coatings, a significant concentration of extrinsic vacancies could give rise to diffusivities orders of magnitude larger than in cases where only intrinsic vacancies are present. The effect of substitutional $\mathrm{Ca}$ in $\mathrm{Yb}$ disilicate on migration barrier energies is currently under investigation.

\section{$\underline{\text { Interstitial diffusion }}$}

The structures of $\beta-\mathrm{Yb}_{2} \mathrm{Si}_{2} \mathrm{O}_{7}, \gamma-\mathrm{Y}_{2} \mathrm{Si}_{2} \mathrm{O}_{7}$ and $\mathrm{HfSiO}_{4}$ are relatively open, containing channels through which interstitial oxygen diffusion might plausibly take place. Such a channel in $\beta$ $\mathrm{Yb}_{2} \mathrm{Si}_{2} \mathrm{O}_{7}$ is shown in figure 2. To investigate this possibility, we have performed migration barrier energy calculations, similar to those described above, for interstitial diffusion. A number of such channels were identified, and stable interstitial points located by relaxing interstitial oxygen atoms initially located along each such channel. Barriers for migration among these stable points were computed using the Improved Dimer method. It should be noted that no attempt was made to identify all stable interstitial points, as the locations of these within, e.g., distorted monoclinic structures can be difficult to determine. For this reason, kMC simulations of interstitial diffusion were not performed, although qualitative information can be obtained from the magnitudes of the migration barrier energies.

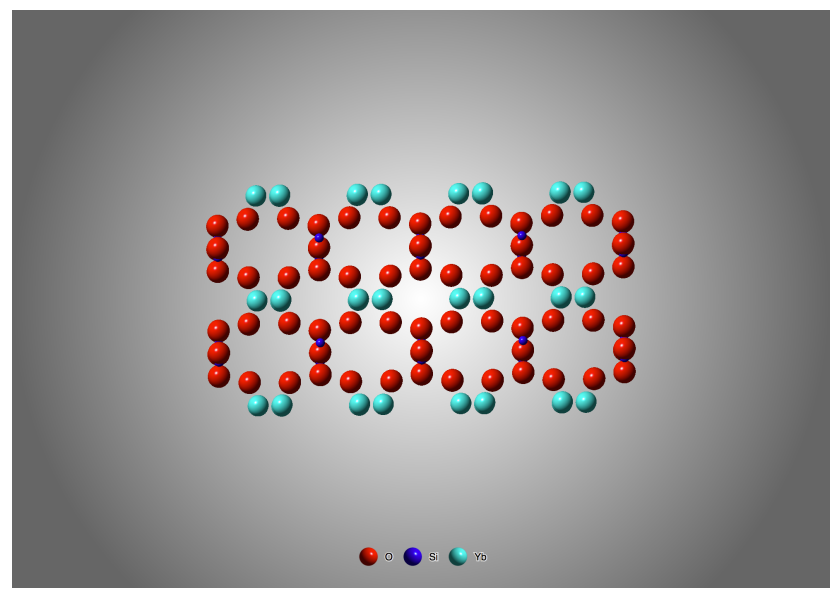

Figure 2. Potential interstitial diffusion channels in $\beta-\mathrm{Yb}_{2} \mathrm{Si}_{2} \mathrm{O}_{7}$

Note that, because the number of interstitial atoms is typically much smaller than the number of interstitial sites, atomic displacements in the kMC simulations can be tracked directly, with no need for a concentration correction factor. Migration barrier energy ranges for all materials except $\delta-\mathrm{Y}_{2} \mathrm{Si}_{2} \mathrm{O}_{7}$ are shown in Table 3. These values are somewhat smaller than those for vacancy diffusion, suggesting that the interstitial oxygen diffusivity will be somewhat larger than the vacancy diffusivity, and much larger than the vacancy-mechanism oxygen diffusivity, which does contain the concentration correction factor. 
The structure of $\delta-Y_{2} \mathrm{Si}_{2} \mathrm{O}_{7}$ is less open than those of the other three materials, and there are few plausible interstitial diffusion paths. Migration barrier calculations for two of those indicate that the barriers are so large that interstitial diffusion will be negligible.

Table 3. Interstitial migration barrier energy ranges.

\begin{tabular}{|c|c|}
\hline Material & Barrier energy range, eV \\
\hline$\beta-\mathrm{Yb}_{2} \mathrm{Si}_{2} \mathrm{O}_{7}$ & $1.03-3.50$ \\
\hline$\gamma-\mathrm{Y}_{2} \mathrm{Si}_{2} \mathrm{O}_{7}$ & $0.56-7.03$ \\
\hline $\mathrm{HfSiO}_{4}$ & $0.66-7.74$ \\
\hline
\end{tabular}

\section{CONCLUSIONS}

Vacancy-mechanism migration barriers for all four materials are large enough that, when only intrinsic vacancies are present, diffusivities from $\mathrm{kMC}$ simulations are very small. On the other hand, if large concentrations of extrinsic vacancies exist, for example, induced by the presence of aliovalent cations, the diffusivities can be orders of magnitude larger. Based on calculations of representative barrier energies, interstitial diffusivities for $\beta-\mathrm{Yb}_{2} \mathrm{Si}_{2} \mathrm{O}_{7}, \gamma-\mathrm{Y}_{2} \mathrm{Si}_{2} \mathrm{O}_{7}$ and $\mathrm{HfSiO}_{4}$ are likely to be large. The structure of $\delta-\mathrm{Y}_{2} \mathrm{Si}_{2} \mathrm{O}_{7}$ is considerably less open, and the representative barriers are large, suggesting that interstitial diffusion will be small.

\section{REFERENCES}

1. K. N. Lee, D. S. Fox and N. P. Bansal, J. Europ. Ceram. Soc. 25, 2005.

2. A. F. Voter, Kinetic Monte Carlo, in Radiation Effects in Solids, Proceedings of the NATO Advanced Study Institute on Radiation Effects in Solids, K. E. Sickafus, E. A. Kotomin, B. P. Uberuaga, eds., 1-23, Springer, Dordrecht, The Netherlands, 2007.

3. W. Kohn, and L. J. Sham, Physical Review 40 (4A): A1133.

4. S. A. Arrhenius, Z. Physik. Chem. 4. 96-116 (1889); P. G. Shewmon, "Diffusion in Solids," pp 43-65, McGraw-Hill, New York, 1963.

5. R. Krishnamurthy, Y.-G. Yoon, D. J. Srolovitz and R. Car, J. Am. Ceram. Soc. 87, 1821 (2004).

6. P. E Blochl, Phys. Review B, 50(24):17953,1994.

7. G. Kresse and D. Joubert, Phys. Rev. B, 59(3):1758, 1999.

8. G. Kresse and J. Hafner, Phys. Rev. B, 47:558, 1993, Rev. B, 49:14251, 1994.G. Kresse and J. Furthmüller, Comput. Mat. Sci., 6:15, 1996, Phys. Rev. B, 54:11169, 1996.

9. J.P. Perdew, J.A. Chevary, S.H. Vosko, K.A. Jackson, M.R. Pederson, D.J. Singh, and C. Fiolhais. Phys. Rev. B, 48:4978, 1993.

10. G. Henkelman and H. Jónsson, J. Chem. Phys. 111, 7010 (1999); A. Heyden, A. T. Bell and F. J. Keil, J. Chem. Phys. 123, 224101 (2005).

11. http://theory.cm.utexas.edu/vtsttools/

12. J. L. Smialek and N. S. Jacobson, 2014. Oxidation of High-Temperature Aerospace Materials. High Temperature Materials and Mechanisms, CRC Press, Boca Raton, FL, pp. 95-162. 